\title{
A Survey of Emission-line Stars in the Outer Part of the Orion Star-forming Region
}

\author{
Suhardja D. Wiramihardja \\ Department of Astronomy and Bosscha Observatory, Bandung Institute \\ of Technology, Indonesia \\ Makoto Nakano \\ Department of Earth Science, Faculty of Education, Oita University, \\ Japan
}

\author{
Tomokazu Kogure \\ Bisei Astronomical Observatory, Bisei-cho, Okayama, Japan
}

\begin{abstract}
We report the result of our survey observations in the outer part of the Orion star-forming region which consists of six Kiso sky areas (150 square degrees). In total, we detected only 48 emission line stars. The low surface density suggests that the Orion star-forming region probably terminates around these areas.
\end{abstract}

\section{Introduction}

Under a cooperative program between Indonesia and Japan we have conducted an extensive survey for emission line stars in the Orion region. A total area of 325 square degrees consisting of 13 Kiso sky areas (Kogure 1994 and references therein) has been surveyed. Here we report the results for outer parts of the region which consist of six Kiso areas, A-0831 $\left(5^{\circ} \times 5^{\circ}\right.$, centred at $\alpha=5^{h} 20^{m}, \delta$ $\left.=+5^{\circ}\right), \mathrm{A}-0832\left(\alpha=5^{h} 40^{m}, \delta=+5^{\circ}\right), \mathrm{A}-0833\left(\alpha=6^{h} 00^{m}, \delta=+5^{\circ}\right), \mathrm{A}-0902$ $\left(\alpha=5^{h} 00^{m}, \delta=+0^{\circ}\right), \mathrm{A}-0905\left(\alpha=6^{h} 40^{m}, \delta=+0^{\circ}\right), \mathrm{A}-0974\left(\alpha=5^{h} 00^{m}, \delta\right.$ $\left.=-5^{\circ}\right)$.

\section{Observations}

The spectral observations were carried out with the $105 / 150 / 330 \mathrm{~cm}$ Kiso Schmidt telescope. The $4^{\circ}$ objective prism attached to the telescope yields a dispersion of $700 \AA \mathrm{mm}^{-1}$ at $\mathrm{H} \alpha$ The plate-filter combinations for our spectral plates are 103aE +RG610. All emulsions were hypersensitised by baking in forming gas. The spectra were not widened. More than 12 plates with exposure times between 10 and 120 minutes were used for this work.

The direct plates in V band were collected using the same telescope with a plate- filter combination of IIaD+GG495. 


\section{Inspection and Measurements}

In order to detect $\mathrm{H} \alpha$ emission stars, all spectral plates were inspected visually using magnifiers. The $\mathrm{H} \alpha$ emission strength relative to the continuum was estimated by eye into six grades of 5 (very strong), 4 (strong), 3 (medium), 2 (weak), 1 (very weak), and 0 (absent or suspected). The numbers of detected $\mathrm{H} \alpha$ emission stars are 4, 3, 6, 3, 12, and 20 for areas A-0831, A-0832, A-0833, A-0902, A-0905, and A-0974, respectively.

The V-band plates were adopted to determine the celestial positions of the detected emission stars. For each plate, at least thirty SAO stars were adopted as standard stars. Measurements were carried out using the XY machine of the Kiso Observatory, and the celestial coordinates were calculated by means of the standard coordinate method. The positional accuracy is estimated to be better than 2 arcsec for each coordinate.

The $\mathrm{V}$ magnitudes of the detected $\mathrm{H} \alpha$ emission stars were derived by adopting the stars of Andrews (1981) as standards and carried out with the iris photometer of the Kiso Observatory. The limiting magnitude is around $\mathrm{V}=17.5$, and the accuracy of measurement is about $0.1 \mathrm{mag}$.

\section{Results}

The apparent magnitudes of the detected $\mathrm{H} \alpha$ emission stars are roughly from $\mathrm{V}=11$ to $\mathrm{V}=17.5$ with a peak around $\mathrm{V}=14-16$, suggesting that the stars are T-Tauri stars. The majority of the $\mathrm{H} \alpha$ intensities are between grades 1 and 3 .

The surface densities of the stars are $0.2,0.1,0.2,0.1,0.5$, and 0.8 per square degree for Kiso areas A-0831, A-0832, A-0833, A-0902, A-0905, and A0974, respectively. These values suggest that the Orion star-forming region probably terminates around these areas.

A full length paper on this work is in preparation.

\section{References}

Andrews A. D., 1981, A Photometric Atlas at the Orion Nebula, (Armagh Obs, Armagh)

Kogure T., 1994, these proceedings

\section{Discussion}

Parker: Have you considered replacing/retaking your plates with Tech Pan film which should not only give you better resolution and $S / N$ in the faint spectra, but which also has a useful sensitivity enhancement at $\mathrm{H} \alpha$ ?

Wiramihardja: At Kiso Observatory, tests to use film instead of glass plate are in an early stage, so film is not available for visiting astronomers. 